\title{
Surfer's Myelopathy: A Radiologic Study of 23 Cases
}

\author{
B.K. Nakamoto, A.M. Siu, K.A. Hashiba, B.T. Sinclair, B.J. Baker, M.S. Gerber, A.M. McMurtray, A.M. Pearce, and J.W. Pearce
}

\section{ABSTRACT}

BACKGROUND AND PURPOSE: Surfing is an uncommon cause of an acute nontraumatic myelopathy. This study describes the MR imaging characteristics and clinical correlates in 23 subjects with surfer's myelopathy.

MATERIALS AND METHODS: This was a retrospective review of 23 cases of surfer's myelopathy from 2003-2012. Spinal cord MR imaging characteristics and neurologic examinations with the use of the American Spinal Injury Association scale were reviewed. Logistic regression was used to determine associations between MR imaging characteristics, American Spinal Injury Association scale, and clinical improvement.

RESULTS: All subjects (19 male, 4 female; mean age, $26.3 \pm 7.4$ years) demonstrated "pencil-like," central T2-hyperintense signal abnormalities in the spinal cord extending from the midthoracic region to the conus with associated cord expansion and varying degrees of conus enlargement on spinal cord MR imaging within 24 hours of symptom onset. $\Pi$ signal was normal. Faint gadolinium enhancement was present in a minority. Although there was a strong correlation between initial American Spinal Injury Association score and clinical improvement $(P=.0032)$, MR imaging characteristics were not associated with American Spinal Injury Association score or clinical improvement.

CONCLUSIONS: Surfer's myelopathy should be considered in the radiographic differential diagnosis of a longitudinally extensive T2-hyperintense spinal cord lesion. MR imaging characteristics do not appear to be associated with severity on examination or clinical improvement.

ABBREVIATIONS: AP $=$ anterior-posterior; ASIA $=$ American Spinal Injury Association

$\mathbf{F}$ irst described in 2004 by Thompson et al, ${ }^{1}$ surfer's myelopathy is a rare, nontraumatic spinal cord injury that usually occurs in novice surfers. A limited number of cases have been reported in the literature. ${ }^{1-10}$ Most of the literature on surfer's myelopathy consists of single case reports. ${ }^{2-9}$ Surfer's myelopathy affects novice surfers who have been lying prone on a surfboard with the lumbar spine hyperextended for prolonged periods of time. These individuals characteristically have a prodrome of back discomfort followed by an acute myelopathy with variable motor-sensory involvement and urinary incontinence. ${ }^{10}$ Given the rarity and novelty of diagnosis, surfer's myelopathy may be underdiagnosed and under-reported. There are no treatments available that are

Received January 30, 2013; accepted after revision March 14.

From the Department of Medicine (B.K.N.), University of Hawaii, Honolulu, Hawaii; Departments of Neurology (B.K.N., A.M.P., J.W.P.), Radiology (B.T.S., B.J.B.), and Neurosurgery (M.S.G.), Straub Clinic and Hospital, Honolulu, Hawaii; Hawaii Pacific Health Research Institute (A.M.S.), Honolulu, Hawaii; Summer Student Research Program (K.A.H.), Hawaii Pacific Health, Honolulu, Hawaii; and Department of Neurology (A.M.M.), Harbor-UCLA Medical Center, Torrance, California.

Please address correspondence to Beau Nakamoto, MD, Straub Clinic and Hospital, Department of Neurology, 888 South King St, Honolulu, HI 96813; e-mail: beau_nakamoto@yahoo.com

三 Indicates article with supplemental on-line table

http://dx.doi.org/10.3174/ajnr.A3599 known to improve clinical outcomes. Recovery ranges from complete return of function to persistent paraplegia.

The etiology of surfer's myelopathy is unknown. Arterial insufficiency from hyperextension of the lumbar spine, venous hypertension associated with obstruction of the inferior vena cava by the liver while lying in a prone position, fibrocartilagenous embolism caused by retrograde embolism of the nucleus pulposus into the radicular spinal cord arteries, or avulsion of perforating arteries have all been proposed. ${ }^{10}$

Surfer's myelopathy is usually associated with a T2-hyperintense spinal cord lesion in the thoracic region, but the imaging findings of this disorder and their clinical correlates have not been carefully characterized. ${ }^{1,10}$ This case series aims to describe the MR imaging characteristics in 23 individuals diagnosed with surfer's myelopathy and to determine if MR imaging characteristics are associated with severity on neurologic examination and clinical outcome.

\section{MATERIALS AND METHODS Identification of Patients}

Subjects were identified by use of the investigators' data base of surfer's myelopathy cases treated or consulted on from 20032012 from 3 community hospitals in the state of Hawaii. Data collected from retrospective chart review included demographics, 

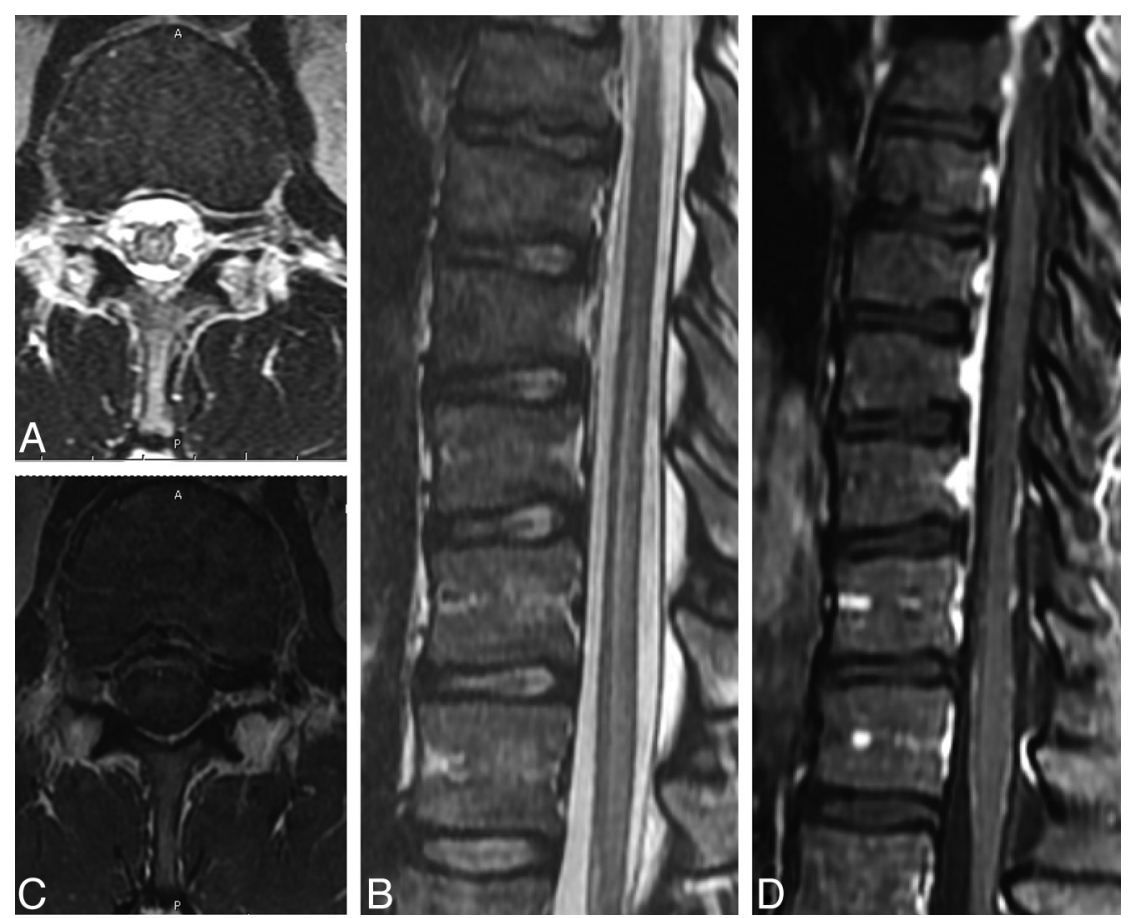

FIG 1. $A$ and $B$, Axial and sagittal T2-weighted images demonstrate mild increased signal in the central aspect of the cord extending to the conus medullaris with cord enlargement. $C$, Axial T1-weighted image demonstrates normal signal intensity. D, Axial T1-weighted postgadolinium image with fat saturation demonstrates faint central cord enhancement.

presenting symptoms, medical/trauma history, American Spinal Injury Association (ASIA) score at admission and follow-up, presence or absence of urinary retention by postvoid residual, and treatment received.

\section{ASIA Score}

The ASIA scoring system is a widely used measurement of functional impairment from spinal cord injury. ${ }^{11}$ The ASIA score is an ordinal scale labeled A-E and categorizes different severities of spinal cord dysfunction. "A" indicates complete spinal cord transection; no motor or sensory function is preserved in the sacral segments S4-S5. "B" describes preserved sensory but no motor function below the level of injury including the sacral segments S4-S5, "C" indicates preservation of motor function with more than half of the muscles being $<3$ on the Medical Research Council scale for testing muscle strength, and " $D$ " is incomplete injury with at least half of the key muscles below the neurologic level being a muscle grade of $\geq 3$. " $E$ " is normal motor and sensory function. ASIA scores were recorded at admission and follow-up after evaluation by the admitting neurologist or neurosurgeon.

\section{Radiologic Assessment}

MR imaging was performed at the treating hospital by use of conventional thoracic and lumbar imaging protocols that included sagittal T1-weighted (TR: 400/TE: 8/NEX: 1/section thickness, $3 \mathrm{~mm}$ at 4-mm intervals), T2-weighted (TR: 3400/TE: 84/ NEX:1.5/section thickness, $3 \mathrm{~mm}$ at 4 -mm intervals), axial T1weighted (TR: 617/TE: 11/NEX: 1/section thickness, $4 \mathrm{~mm}$ at 5-mm intervals), and T2-weighted fast spin-echo (TR: 4283/TE: 99/NEX: 2/section thickness, $4 \mathrm{~mm}$ at 5-mm intervals) sequences on a 1.5T GE Signa (Milwaukee, Wisconsin) or Phillips Achieva
(Andover, Massacjusetts) scanner. Sagittal STIR sequence (TR: 3100/TE: 39/NEX: $1 /$ section thickness, $3 \mathrm{~mm}$ at 4 -mm intervals) was also used in 5 subjects. Intravenous gadopentetate dimeglumine $(0.2$ $\mathrm{mL} / \mathrm{kg}$ ) contrast was administered at the discretion of the radiologist at the time of initial evaluation. MR imaging characteristics were reviewed by 2 radiologists with a Certificate of Added Qualification in neuroradiology. Spinal cord MR imaging characteristics reviewed included T1-signal intensity, extent of continuous T2-signal abnormality on sagittal imaging, location of T2-signal abnormality on axial sections, number of involved vertebral body levels, continuous T2-signal abnormality length, degree of T2-signal abnormality, presence/absence of cord expansion, anterior-posterior (AP) conus diameter, and the presence of gadolinium enhancement when performed. Subtle T2-signal abnormalities in the spinal cord were defined as mild (Fig 1). Obvious T2signal abnormalities were defined as moderate (Fig 2), and T2-signal abnormalities in the spinal cord that were the same as CSF intensity were defined as severe (Fig 3). T2 signal of the vertebral bodies was also assessed.

\section{Statistical Methods}

Statistical analysis was performed with the use of STATA/IC11.1 for Windows (StataCorp, College Station, Texas). Standard descriptive statistics were used to summarize clinical and MR imaging characteristics. Logistic regression was used to analyze the relationship between ASIA scores as the dependent variable and MR imaging characteristics (ie, number of vertebral body levels of involvement, continuous T2-signal abnormality length, degree of T2-signal abnormality, presence/absence of cord expansion, and AP diameter of conus) as the independent variables. ASIA score on admission and on follow-up were analyzed separately against MR imaging characteristics. Grouped ASIA scores (A and B, C-E) on admission and follow-up were created and analyzed against MR imaging characteristics. The degree of T2-signal abnormalities was recorded as an ordinal categoric variable (ie, mild, moderate, or severe). Fisher exact test was used for categoric variables when appropriate. Variables that were not normally distributed were analyzed by means of the nonparametric Kruskal-Wallis and Mann-Whitney tests. Logistic regression was used to analyze the relationship between clinical improvement as the dependent variable and MR imaging characteristics and treatment received as the independent variables. Clinical improvement was defined as an improvement of $\geq 1$ ASIA grade from admission to follow-up. Spearman correlation was used with ordinal and nonparametric variables.

A determination of exemption from Institutional Review Board requirements and a waiver of Health Insurance Portability 

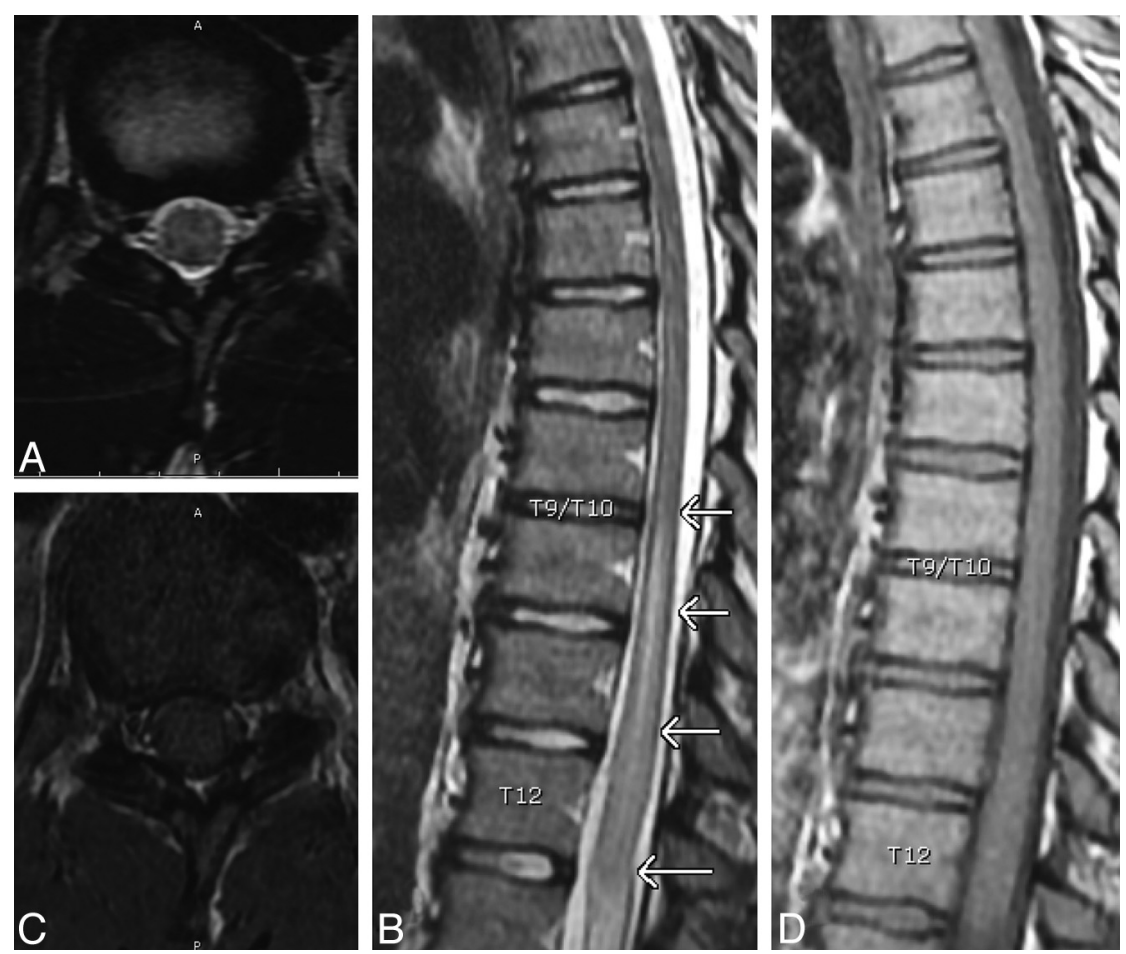

FIG 2. $A$ and $B$, Axial and sagittal T2-weighted images demonstrate moderate increased signal in the central aspect of the cord extending to the conus medullaris with cord enlargement. $C$ and $D$, Axial and sagittal T1-weighted images demonstrate normal signal intensity.
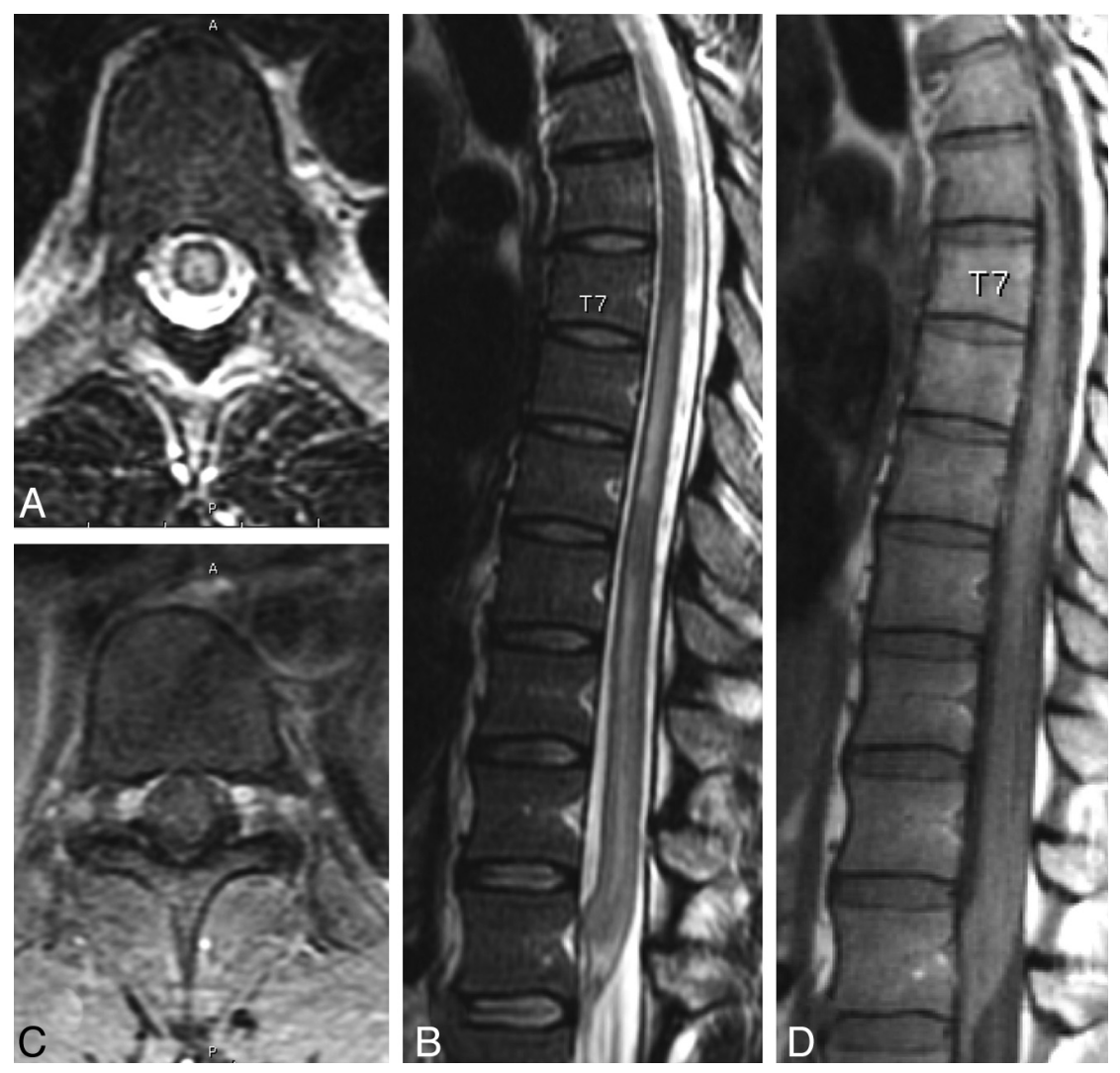

C
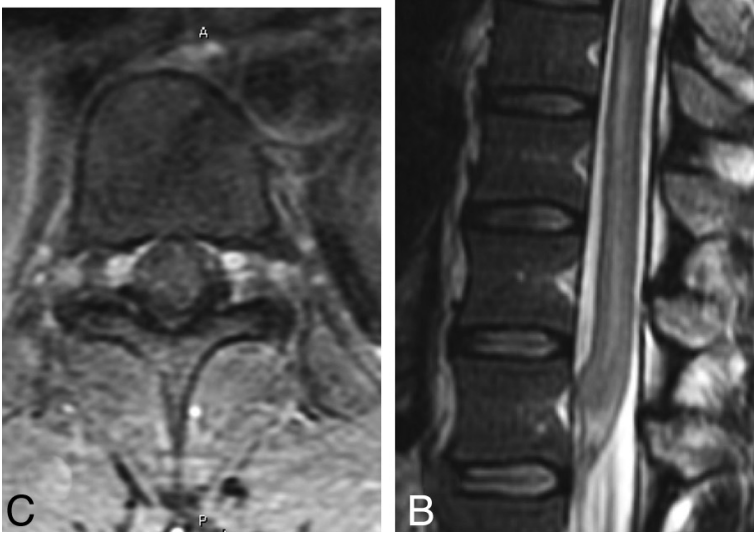

FIG 3. $A$ and $B$, Axial and sagittal T2-weighted images demonstrate severe increased signal in the central aspect of the cord extending to the conus medullaris with cord enlargement. $C$ and $D$, Axial and sagittal T1-weighted images demonstrate normal signal intensity. and Accountability Act authorization were obtained for this retrospective chart review.

\section{RESULTS}

\section{Clinical Characteristics}

A total of 24 cases were identified. Twenty-three cases (19 male, 4 female; mean age, $26.3 \pm 7.4$ years) had both clinical data and MR imaging data available for review. Race/ethnicity was equally distributed between Asian (11) and white (11), with 1 case reporting mixed race. All subjects were healthy individuals without a preceding systemic illness. Twenty-two subjects $(22 / 23)$ presented with the acute low back pain associated with varying degrees of paraparesis while surfing. Fifteen subjects (15/23) presented with an ASIA score of $\mathrm{C}$ or better. On admission, 5 subjects had an ASIA score of A, 3 subjects had an ASIA score of B, 5 subjects had an ASIA score of C, 9 subjects had an ASIA score of D, and 1 subject presented with an ASIA score of E. Sixteen subjects had a sensory level ranging from T8-L3, whereas 6 subjects had a normal sensory examination. Sensory examination was not performed for 1 subject. Twenty-two subjects (22/23) had urinary retention on postvoid residual on admission. No subject reported a history of trauma. Seven subjects were treated with a full course of intravenous methylprednisolone on the basis of the Third National Acute Spinal Cord Injury Study protocol for acute spinal cord injury (ie, an intravenous bolus dose of methylprednisolone $30 \mathrm{mg} / \mathrm{kg}$ over 15 minutes, followed by a 45 -minute pause, and then a 23-hour continuous intravenous infusion of $5.4 \mathrm{mg} / \mathrm{kg}$ per hour). ${ }^{12}$

Most patients included in this review were visitors to Hawaii and transferred to hospitals in their home location for additional follow-up and treatment. Follow-up data, therefore, were limited to outcome at discharge for many of the cases. Duration of follow-up ranged from 1 day to 8 years (median, 6 days; interquartile range, 3-14 days).

\section{MR Imaging Characteristics}

All MR imaging studies were performed within 24 hours of symptom onset. Further details regarding time between symptom onset and time when the MR imaging was performed were unavailable. Follow-up MRI was not per- 
formed. Refer to the On-line Table for individual patient MR imaging characteristics. All subjects had "pencil-like" longitudinally extensive hyperintense T2-weighted signal abnormalities extending from the midthoracic region to the conus with associated cord expansion and varying degrees of conus enlargement (mean, $8.4 \pm 0.8 \mathrm{~mm}$; range, 7-10) on sagittal sequences, and these were located in the central cord on axial sequences. The cranial extent of continuous T2-signal abnormality ranged from T5-T10. The T2-signal abnormality extended down to the conus in all subjects. The number of vertebral body levels of involvement ranged from 3-7.5 levels (mean, $5.1 \pm 1.4$ ). The mean length of T2-signal abnormality was $13.8 \pm 3.1 \mathrm{~cm}$ (range, 9-21). Degree of T2-signal hyperintensity ranged from mild (6/23, Fig 1$)$, to moderate (7/23, Fig $2)$, to severe (10/23, Fig 3$)$. The spinal cord was normal on T1-weighted sequences. No intramedullary hemorrhage was present. Gadolinium enhancement ranged from no gadolinium enhancement (7/11) to trace gadolinium enhancement (4/11). No other radiologic abnormalities were present. No subject had central canal stenosis, disk prolapse or herniation at the levels of T2-signal abnormalities, syrinx, fractures, dislocations, ligamentous injuries, hematomas, or perimedullary or intramedullary vascular dilations. T2-signal of the vertebral bodies was normal in all subjects. DWI sequences were not performed. No subject had evidence of inferior vena cava compromise.

\section{Association Between MR Imaging Characteristics and ASIA Score on Admission and Follow-Up}

The relationship between MR imaging characteristics (degree of T2-signal abnormality, $P=.86$; number of vertebral body levels of involvement, $P=.51$; length of continuous T2-signal abnormality, $P=.25$; AP diameter of conus, $P=.45$ ) and ASIA score on admission showed no associations when tested with the Fisher exact test. Similarly, there were no associations between MR imaging characteristics (degree of T2-signal abnormality, $P=.42$; number of vertebral body levels of involvement, $P=.28$; length of continuous T2-signal abnormality, $P=.57$; AP diameter of conus, $P=.99$ ) and ASIA follow-up score. There were no statistically significant associations between grouped ASIA scores on admission or follow-up and MR imaging characteristics.

\section{Association Between MR Imaging Characteristics and Clinical Improvement}

The median follow-up time was 6 days (interquartile range, 3-14 days). Approximately $65 \%$ of the cases showed no improvement in ASIA score from admission (13/20). Clinical improvement was not significantly associated with any MR imaging characteristics (degree of T2-signal abnormality, $P=.20$; number of vertebral body levels of involvement, $P=.96$; length of continuous T2signal abnormality, $P=.51$; AP diameter of conus, $P=.79$ ) when tested with logistic regression. Follow-up time was not correlated with follow-up ASIA score $(P=.67$, Kruskal-Wallis) or grouped follow-up ASIA score ( $P=.23$, Mann-Whitney).

\section{Association Between ASIA Score on Admission and Follow-Up}

There was a strong correlation between ASIA score on admission and clinical improvement $(P=.0079$, Spearman $)$. All of the patients who presented with an ASIA score of A or B on admission showed no improvement at follow-up (7/20). Grouped ASIA scores on admission showed a strong association with clinical improvement $(P=0.22$, Fisher exact). Follow-up time $(P=.684$, logistic regression) and treatment with intravenous methylprednisolone $(P=.613$, Fisher exact $)$ were not significantly associated with improvement.

\section{DISCUSSION}

\section{Study Significance}

This is the largest case series to date detailing the radiologic characteristics of surfer's myelopathy. Little attention has been given to MR imaging characteristics and their clinical correlates in previous case series of this rare disorder. ${ }^{10}$

\section{Radiologic Characteristics of Surfer's Myelopathy}

In our study, spinal cord MR imaging demonstrates a longitudinally extensive region of hyperintense T2-signal abnormality extending from the midthoracic region to the conus with associated cord expansion and variable degrees of fusiform enlargement of the conus on sagittal sequences. The hyperintense signal on T2weighted sequences usually extends cranially to the midthoracic region, but the region of involvement can be as short as from the conus to $\mathrm{T} 10$ or as far cranially as $\mathrm{T} 5$. The $\mathrm{T} 2$ abnormality involves the central cord on axial sequences. Spinal cord signal is normal on T1-weighted sequences with either absent or trace regions of gadolinium enhancement. Our findings are consistent with previous descriptions of surfer's myelopathy with the exception that others have also reported sparing of the conus in addition to enhancement of the ventral roots of the cauda equina. ${ }^{1,4,10}$ Although T1-weighted noncontrast sequences are usually normal, patchy increased T1-weighted signal changes on initial presentation and repeat imaging 1 month later, presumably from petechial hemorrhage, have rarely been described. ${ }^{2,6}$ The T1-weighted signal of the spinal cord was normal in all subjects in our study, and no subject had evidence of intramedullary hemorrhage. The trace gadolinium enhancement seen in the spinal cords of 4 subjects in our study and the rare case reports of petechial hemorrhage in other studies ${ }^{2,6}$ are consistent with the slight inflammatory and hemorrhagic changes demonstrated on CSF analysis in individuals with surfer's myelopathy in whom a lumbar puncture was performed. ${ }^{10}$

The evolution of the MR imaging findings associated with surfer's myelopathy has not been well described because of the absence of consistent serial imaging and long-term follow-up in reported cases. Five published papers detailed the time between symptom onset and imaging, and conventional spinal cord MR imaging was abnormal within $2-12$ hours. ${ }^{1,2,4,5,8}$ One case reported by Kelly and Wright ${ }^{3}$ was initially normal but was subsequently abnormal on repeat imaging within 24 hours of the initial MR imaging. ${ }^{3}$ DWI with ADC mapping has inconsistently demonstrated restricted diffusion in cases of surfer's myelopathy. ${ }^{10}$ 
In the acute setting, the abnormal T2-hyperintensity severity can increase and extend rostrally up to 4 vertebral body segments. ${ }^{1,2}$ The abnormal T2-signal has been shown to resolve spontaneously in some studies and improve when subjects are treated with steroids in other studies. ${ }^{1,4,7}$ Descriptions of chronic MR imaging changes in surfer's myelopathy have not been reported.

\section{Radiologic Differential Diagnosis}

A characteristic history aids in narrowing the radiographic differential diagnosis to surfer's myelopathy. These individuals are young, inexperienced surfers who lay prone on a surfboard for a prolonged period and present with nontraumatic acute onset of back pain followed by symptoms of a myelopathy. Other radiographic considerations for a longitudinally extensive hyperintense spinal cord lesion on T2-weighted images associated with an acute intramedullary myelopathy have been reviewed elsewhere and include an idiopathic transverse myelitis, MS, longitudinally extensive transverse myelitis, venous hypertension associated with spinal dural arteriovenous fistula, and arterial infarct. ${ }^{13-16}$ Idiopathic transverse myelitis, MS, and longitudinally extensive transverse myelitis are all acute inflammatory spinal cord syndromes that can be grouped under the umbrella term "transverse myelitis." Whereas there is a great deal of overlap in imaging appearances of these conditions, there are distinct imaging appearances that are helpful for differentiation. Idiopathic transverse myelitis, which is usually preceded by a viral illness or vaccination, has low signal intensity on T1-weighted images and high signal intensity on T2-weighted images. The spinal cord caliber can be normal or mildly enlarged, affecting the cervical and/or thoracic cord, and may have patchy or peripheral gadolinium enhancement. MS plaques but are peripherally located, typically involve the dorsolateral regions of the cord, and can also have brain involvement. Most MS spinal lesions are smaller than 2 vertebral body segments. In contrast, idiopathic transverse myelitis typically extends 3 to 4 vertebral segments in length, and longitudinally extensive transverse myelitis is a contiguous spinal cord lesion extending over $\geq 3$ vertebral segments. ${ }^{13}$ Neuromyelitis optica, or Devic syndrome, is one cause of longitudinally extensive myelitis that can also have an associated optic neuritis. Whereas all these conditions can be considered in the radiographic differential diagnosis, the characteristic contiguous, longitudinally extensive midthoracic to conus, central cord involvement, and historical correlate differentiate these entities from surfer's myelopathy.

The radiographic findings of surfer's myelopathy can be difficult to differentiate from venous hypertension and an arterial spinal cord infarct. Venous hypertension associated with a spinal dural arteriovenous fistula can be distinguished by the presence of dilated, tortuous perimedullary vessels seen as flow voids dorsally in the thecal sac and by the history of a chronic progressive myelopathy in contrast to the acute onset of symptoms after surfing in those with surfer's myelopathy. In our study, the radiologic characteristics on spinal cord MR imaging may be indistinguishable from an arterial thoracic spinal cord infarct.

\section{Pathogenesis}

Although no definite histopathologic confirmation has been published in this population, surfer's myelopathy probably is the result of a vascular phenomenon involving dynamic compression, vasospasm, or thrombotic infarction of the artery of Adamkiewicz, which occurs as the result of hyperextension of the spinal cord while individuals are lying prone on a surfboard. ${ }^{10}$ The MR imaging characteristics in our study support the hypothesis that surfer's myelopathy is the result of spinal cord infarction in the vascular distribution of the artery of Adamkiewicz. Unlike the cerebral vessels, the spinal arteries run along a mobile structure, making them prone to mechanical damage as may occur with prolonged hyperextension of the back during surfing. The thoracic spinal cord is at a greatest risk of ischemia as a result of poor collateral vascular supply in this region and is consistent with the variable levels of thoracic spinal cord involvement demonstrated in our study. ${ }^{17}$ The variable levels of thoracic spinal cord involvement may be explained by the variable origins of the artery of Adamkiewicz, arising from T5-T8 in 15\%, T9-T12 in 75\%, and L1-L2 in $10 \%$ of the population. ${ }^{17}$ The central cord involvement seen on MR imaging in all of our subjects may be explained by the small central vascular supply of the thoracic spinal cord, and, because the anterior and posterior spinal arteries run along the surface of the cord, the central cord is also a watershed region. A few studies have demonstrated increased diffusion restriction on DWI correlating with decreased signal on ADC mapping in surfer's myelopathy, supporting the hypothesis that the T2-signal abnormalities represent cytotoxic edema in the setting of an acute spinal cord infarction. ${ }^{8,10}$ Conventional spinal angiograms were normal when performed in previously described cases. ${ }^{1,5,10}$

\section{Clinical Correlates}

In our study, MR imaging characteristics were not associated with severity of the myelopathy as measured by the ASIA score and did not predict clinical improvement on follow-up. In a case series of 19 subjects, Chang et al ${ }^{10}$ also reported that restricted diffusion on DWI with ADC mapping did not correlate with outcome. This analysis, however, was performed on subset of 10 patients in the case series who had DWI with ADC mapping, of whom 6 showed restriction. Our finding that ASIA score on admission predicts clinical improvement is consistent with those reported by Chang et al. ${ }^{10}$ Treatment with high-dose intravenous methylprednisolone in 7 subjects in our study was not associated with clinical improvement, but this probably was a result of our small sample size.

\section{Limitations}

Although our case series is the largest to date, our sample size may have been underpowered to detect a relationship between MR imaging characteristics, disease severity, and clinical improvement. Second, all subjects were not imaged with the same MR imaging protocol at standard times, which may have increased the variability of the abnormal T2-signal intensity, extent of involvement, and AP diameter of the conus on obtained images. DWI with ADC mapping was not obtained in our study, and the role of this sequence in the clinical evaluation of spinal 
cord ischemia and surfer's myelopathy remains unclear. Third, lack of consistent long-term follow-up limits characterization of chronic radiographic characteristics of surfer's myelopathy. Finally, confirmatory histopathology to determine that surfer's myelopathy is a result of an arterial infarction was not feasible.

\section{CONCLUSIONS}

In summary, surfer's myelopathy is a rare form of an acute myelopathy, which should be considered in the radiographic differential diagnosis of a longitudinally extensive T2-hyperintense spinal cord lesion in the context of a surfing history. Greater awareness of the characteristic history associated with this disorder is important to making the correct radiographic diagnosis. Severity of the radiographic characteristics of surfer's myelopathy is not associated with severity on initial examination or clinical improvement.

\section{ACKNOWLEDGMENTS}

We thank our study participants and their families for their roles in this study.

Disclosures: Beau Nakamoto—RELATED: Grant: Research support from National Institutes of Health (grant U54MD007584)* ( ${ }^{*}$ money paid to institution).

\section{REFERENCES}

1. Thompson TP, Pearce J, Chang G, et al. Surfer's myelopathy. Spine 2004;29:E353-56

2. Aviles-Hernandez I, Garcia-Zozaya I, DeVillasante JM. Nontraumatic myelopathy associated with surfing. I Spinal Cord Med 2007;30:288-93

3. Kelly M, Wright K. A case of surfers' myelopathy. Am J Clin Med 2010;7:74-75

4. Dhaliwal PP, Cenic A, Eesa M, et al. An unusual case of myelopathy: surfer's myelopathy. Can J Neurol Sci 2011;38:354-56

5. Chung HY, Sun SF, Wang JL, et al. Non-traumatic anterior spinal cord infarction in a novice surfer: a case report. J Neurol Sci 2011;302:118-20

6. Shuster A, Franchetto A. Surfer's myelopathy: an unusual cause of acute spinal cord ischemia: a case report and review of the literature. Emerg Radiol 2011;18:57-60

7. Karabegovic A, Strachan-Jackman S, Carr D. Surfer's myelopathy: case report and review. Canadian Journal of Emergency Medicine 2011;13:357-60

8. Lieske J, Cameron B, Drinkwine B, et al. Surfer's myelopathy: demonstrated by diffusion-weighted magnetic resonance imaging: a case report and literature review. J Comput Assist Tomogr 2011;35:492-94

9. Lin $\mathrm{CY}, \mathrm{Fu} \mathrm{JH}, \mathrm{Li} \mathrm{SC}$, et al. Surfer's myelopathy. QJM 2012;105:373-74

10. Chang CW, Donovan DJ, Liem LK, et al. Surfers' myelopathy: a case series of 19 novice surfers with nontraumatic myelopathy. Neurology 2012;79:2171-76

11. Reference Manual of the International Standards for Neurological Classification of Spinal Cord Injury. Chicago: American Spinal Injury Association; 2003

12. Bracken MB, Shepard MJ, Holford TR, et al. Administration of methylprednisolone for $\mathbf{2 4}$ or $\mathbf{4 8}$ hours or tirilazad mesylate for $\mathbf{4 8}$ hours in the treatment of acute spinal cord injury: results of the Third National Acute Spinal Cord Injury Randomized Controlled Trial: National Acute Spinal Cord Injury Study. JAMA 1997;277:1597-604

13. Do-Dai DD, Brooks MK, Goldkamp A, et al. Magnetic resonance imaging of intramedullary spinal cord lesions: a pictorial review. Curr Probl Diagn Radiol 2010;39:160-85

14. Bou-Haidar P, Peduto AJ, Karunaratne N. Differential diagnosis of T2 hyperintense spinal cord lesions: part B. J Med Imaging Radiat Oncol 2009;53:152-59

15. Sheerin F, Collison K, Quaghebeur G. Magnetic resonance imaging of acute intramedullary myelopathy: radiological differential diagnosis for the on-call radiologist. Clin Radiol 2009;64:84-94

16. Kitley JL, Leite MI, George JS, et al. The differential diagnosis of longitudinally extensive transverse myelitis. Mult Scler 2012;18: 271-85

17. Shamji MF, Maziak DE, Shamji FM, et al. Circulation of the spinal cord: an important consideration for thoracic surgeons. Ann Thorac Surg 2003;76:315-21 